\title{
Acute pancreatitis in association with small cell lung carcinoma: potential pitfall in diagnosis and management
}

\author{
Simon G. Allan ${ }^{1}$, Nigel Bundred ${ }^{2}$, Oleg Eremin ${ }^{2}$ and Robert C.F. Leonard ${ }^{1}$ \\ ${ }^{1}$ University Department of Clinical Oncology, Western General Hospital, Edinburgh EH4 $2 X U$ and ${ }^{2}$ University \\ Department of Clinical Surgery, Royal Infirmary, Edinburgh EH3 9YW, UK.
}

\begin{abstract}
Summary: Tumour metastases to the pancreas are a rare but recognized cause of acute pancreatitis. there is a $\mathbf{2 4 - 4 0 \%}$ incidence of pancreatic involvement from small cell lung cancer in autopsy series but only a very few cases of tumour-induced acute pancreatitis have been described. Chemotherapy has been advocated as the primary therapy in patients with known oat cell carcinoma who develop acute pancreatitis. We describe 2 patients with acute haemorrhagic pancreatitis in association with disseminated small cell carcinoma but without evidence of tumour invasion in the gland and with gall stones present in the gall bladder. Chemotherapy would have been inappropriate therapy for these patients.
\end{abstract}

\section{Introduction}

Several tumours, including breast, prostate and kidney, have been reported to metastasize to the pancreas (Cameron-Strange, 1983; Gatchell \& Minor, 1972; Levine \& Danovitch, 1973; Line \& Dealey, 1971; McLatchie \& Imrie 1981) but acute pancreatitis caused by metastatic tumour is rare. Bronchochogenic carcinoma (particularly small cell carcinoma) may be the commonest metastatic tumour to induce actue pancreatitis (Levine \& Danovitch, 1973; Niccolini et al., 1976; Yeung et al., 1979). We report 2 patients with known small cell carcinoma of the lung and acute pancreatitis and comment on the danger of assuming that the pancreatitis is secondary to tumour metastases.

\section{Case reports}

Case 1

A 73 year old woman was admitted as a surgical emergency giving a 2 day history of persistent abdominal pain radiating to her lower thoracic spine. She was constipated and vomiting. She had received local radiotherapy for small cell carcinoma of the left lung 3 months previously. Physical examination revealed generalized abdominal tenderness, guarding and absent bowel sounds. Auscultation of the chest revealed poor air entry to the left apex with bilateral, and coarse basal creptitations. Her blood urea and

Correspondence: S.G. Allan M.B., M.R.C.P.

Accepted: 25 October 1984 electrolytes were normal, amylase $3840 \mathrm{U} / 1$ (normal $50-300 \mathrm{U} / \mathrm{l}$ ), calcium $2.31 \mathrm{nmol} / 1$ (normal $2.25-2.5$ $\mathrm{mmol} / \mathrm{l})$. A diagnosis of acute pancreatitis with bronchopneumonia was made. She was treated by nasogastric suction, intensive intravenous therapy, antibiotics and oxygen. Her condition, however, deteriorated and she died on the fifth day after admission. Autopsy revealed numerous flecks of fat necrosis throughout the abdomen with haemorrhagic oedema of the pancreas. Multiple sections of the pancreas revealed fat necrosis, local congestion and marked inflammatory infiltrate without evidence of tumour. The gall bladder contained a few small calculi but the biliary and pancreatic ducts were patent. Small cell carcinoma was present in the left main bronchus with nodal metastases in the thorax and multiple hepatic metastases. There was no pathological evidence of pneumonia.

Case 2

Following chemotherapy for limited small cell lung carcinoma, a 67 year old man had been in remission for 6 months. At this point he suddenly became unwell and presented with a 1 week history of blurred vision. Computed tomography of the brain revealed cerebral metastases and he was commenced on dexamethasone. He did not respond to treatment and became increasingly drowsy. In the 2 days before his death (10 d after admission), the patient complained of severe central abdominal pain with epigastric tendernesss on examination. He received symptomatic therapy for dyspepsia secondary to his steroid therapy 
but died. His blood urea was $7.2 \mathrm{mmol} / \mathrm{l}$ pre-mortem, with normal electrolytes and calcium but a serum amylase was not obtained.

Autopsy revealed a gross necrotizing pancreatitis with marked auto-digestion and an intense inflammatory infiltrate. There was no microscopic evidence of metastatic tumour in the pancreas and the biliary tree was free of gall stones or obstruction although there were 3 small stones present in the gall bladder. Extensive small cell carcinoma metastases were seen in adrenal glands, kidneys, liver, thyroid, brain, bone marrow and scattered nodes in the porta hepatis but without evidence of biliary obstruction.

\section{Discussion}

Acute pancreatitis is a serious condition carrying a mortality of $25-40 \%$, with common aetiological factors including gall stones and alcohol (Anonymous, 1981; Taylor, 1977). Acute pancreatitis associated with non-pancreatic neoplasia is rare. Four papers (Levine \& Danovitch, 1973; McLatchie \& Imrie, 1981; Niccolini et al., 1976; Yeung et al., 1979) have described acute pancreatitis caused by lung carcinomas, Yeung et al. (1979) proposed that the pancreatitis, in association with small cell lung cancer,

\section{References}

ANONYMOUS (1981). Pancreatitis. In Bailey \& Love's Short Practice of Surgery, revised by Rains, A.J.H. \& Ritchie, H.D. p. 940. H.K. Lewis: London.

CAMERON-STRANGE, A. (1983). Acute pancreatitis associated with lymphosarcoma. British Journal of Surgery, 70, 444.

GATCHELL, F.G. \& MINOR, D. (1972). Malignant melanoma of the eye, metastatic after 29 years. Journal of the Oklahoma State Medical Association, 65, 211.

LEVINE, M. \& DANOVITCH, S. (1973). Metastatic carcinoma to the pancreas. American Journal of Gastroenterology, 60, 290.

LINE, O.H. \& DEALEY, T.J. (1971). The necropsy findings in carcinoma of the bronchus. British Journal of Diseases of the Chest, 65, 238. may result from ductal obstruction following direct invasion or peripancreatic compression due to metastatic enlargement of regional lymph nodes. In this same series the authors proposed that metastaticinduced acute pancreatitis is not a rare clinical entity in patients with small cell lung cancer and that intensive polychemotherapy should be promptly initiated in such patients. They agree that diagnosis of metastatic invasion of the pancreas is very difficult without an exploratory laparotomy and suggest that chemotherapy be employed if conventional measures have not succeeded.

Our two patients had acute necrotizing pancreatitis with evident dissemination of tumour at autopsy. Neither patient, however, had any evidence of tumour infiltration of the pancreas nor any nodal obstruction of the pancreatic or biliary ducts. Both patients did, however, have small gall stones present in their gall bladders and the strong likelihood is that cholelithiasis was responsible for the onset of pancreatitis. Chemotherapy would have been inappropriate therapy for either of these patients. Acute pancreatitis caused by metastatic small cell lung cancer is rare and it behoves the clinician to exclude the common causes of acute pancreatitis before considering intensive chemotherapy.

MCLATCHIE, G.R. \& IMRIE, C.W. (1981). Acute pancreatitis associated with tumour metastases in the pancreas. Digestion, 21, 13.

NICCOLINI, D.G., GRAHAM, J.H. \& BANKS, P.A. (1976). Tumour-induced acute pancreatitis. Gastroenterology, 71, 142.

TAYLOR, J.H. (1977). An aetiological and therapeutic review of acute pancreatitis. British Journal of Hospital Medicine, $18,546$.

YEUNG, K.J., HAIDAK, D.J., BROWN, J.A. \& ANDERSON, D. (1979). Metastatic-induced acute pancreatitis in small cell bronchogenic carcinoma. Archives of Internal Medicine, 139, 552. 